UCI-TR-2005-30

gr-qc/0507039

\title{
The Newtonian limit at intermediate energies
}

\author{
J. A. R. Cembranos \\ Department of Physics and Astronomy, University of California, Irvine, CA 92697 USA
}

(Dated: October 9, 2018)

\begin{abstract}
We study the metric solutions for the gravitational equations in Modified Gravity Models (MGMs). In models with negative powers of the scalar curvature, we show that the Newtonian Limit (NL) is well defined as a limit at intermediate energies, in contrast with the usual low energy interpretation. Indeed, we show that the gravitational interaction is modified at low densities or low curvatures.
\end{abstract}

PACS numbers: 04.50.+h, 04.25.Nx, 98.80.-k, 95.35.+d

Einstein's General Relativity (GR) describes the gravity field in a very successful way by the metric tensor of the space-time through the Einstein-Hilbert (EH) action. However, this action is non renormalizable and many authors have tried to solve different cosmological and astrophysical puzzles by modifying it, i.e. with MGMs; for example, the introduction of Lagrange densities proportional to $R^{n}$ with $n>1$ (Large MGMs or LMGMs) leads to Starobinsky inflation [1]. In the last years, terms proportional to $R^{n}$ with $n<1$ (Small MGMs or SMGMs) have been taken into account to explain the present cosmic acceleration without the typical cosmological constant $(n=0)$ or dark energy [2]. In early times, it is reasonable that terms with $n>1$ can be significant but negligible today; whereas terms with $n<1$ can be neglected in the early Universe but not in the present or late epochs, when the space-time curvature is very small.

The introduction of new scalar curvature dependent terms in the action modifies Einstein's Equations (EEs) in the following way:

$$
\begin{aligned}
& {\left[1+\varepsilon^{\prime}(R)\right] R_{\mu \nu}-\frac{1}{2}[R+\varepsilon(R)] g_{\mu \nu}} \\
& +\mathcal{I}_{\alpha \beta \mu \nu} \nabla^{\alpha} \nabla^{\beta}\left[\varepsilon^{\prime}(R)\right]=\frac{T_{\mu \nu}}{M_{\mathrm{Pl}}^{2}},
\end{aligned}
$$

where $\mathcal{I}_{\alpha \beta \mu \nu} \equiv\left(g_{\alpha \beta} g_{\mu \nu}-g_{\alpha \mu} g_{\beta \nu}\right)$ and $\varepsilon^{(n)}(R)$ is the $\mathrm{n}^{\text {th }}$ derivative of $\varepsilon(R)$ with respect to the curvature. We are supposing a small modification of the $\mathrm{EH}$ action:

$$
S_{\mathrm{G}}=\frac{1}{2} \int d^{4} x \sqrt{-g} M_{\mathrm{P} 1}^{2}[R+\varepsilon(R)]
$$

with the reduced Planck mass $M_{\mathrm{Pl}}=\left(8 \pi G_{N}\right)^{-1 / 2} \simeq$ $2.4 \times 10^{18} \mathrm{GeV}$ (we are adopting the convention: $\hbar=$ $c=1)$. We are focusing on SMGMs of the form:

$$
\varepsilon(R)=\lambda \mu^{2-2 n} R^{n}, \quad \lambda= \pm 1, \quad n<1(n \neq 0) .
$$

However, other terms present a similar behavior (as a logarithmic one) and some results of this work are general for any $\varepsilon(R)$. To the above gravitational action, we have to add the standard matter one:

$$
S_{\mathrm{M}}=\int d^{4} x \sqrt{-g} \mathcal{L}_{\mathrm{M}} .
$$

In the case that there is no matter, due to the symmetries of the vacuum, the solutions are maximally symmetric space-times with a constant curvature scalar $R_{0}$, which is solution of the following equation:

$$
R_{0}\left[1-\varepsilon_{0}^{\prime}\right]+2 \varepsilon_{0}=0,
$$

where $\varepsilon_{0}^{(n)} \equiv \varepsilon^{(n)}\left(R_{0}\right)$. It is interesting to note that without a cosmological constant, $R_{0}=0$, is not in general a solution for SMGMs. In fact, a large number of works have studied such modifications with $\mu \sim H_{0} \sim 10^{-33} \mathrm{eV}$ to explain the observed cosmic acceleration [2, 3, 4, [5, 6], whose origin could come from different compactifications of extra dimensions in M-theory [7].

On the other hand, the third term in the left hand side of (11) can produce important instabilities 8], whose solution seems to demand the introduction of higher order terms $(n>1)$ in the scalar curvature [3]. However, in the present literature, it is possible to find three other problems of SMGMs which are directly related to our present discussion [9, 10, 11]. In this work we show that these three problems are connected and that their analyses are not enough to exclude SMGMs. On the contrary, we argue that this type of models presents a well defined NL at intermediate scales.

The NL in SMGMs was studied in 11] through weak field expansions around maximally symmetric vacuum solutions. It means that we can perform a series in the $\varepsilon(R)$ function supposing analyticity in the background solution $R_{0}: \varepsilon(R)=\varepsilon_{0}+\varepsilon_{0}^{\prime}(\delta R)+\varepsilon_{0}^{\prime \prime}(\delta R)^{2} / 2+\ldots$ with $\delta R \equiv R-R_{0}$. For instance, taking trace in (1) we obtain a covariant expansion, whose zero order is given by (5) and the first order by:

$$
\left[1+\varepsilon_{0}^{\prime}-R_{0} \varepsilon_{0}^{\prime \prime}\right](\delta R)-3 \varepsilon_{0}^{\prime \prime} \nabla^{\alpha} \nabla_{\alpha}(\delta R)=-\frac{T}{M_{\mathrm{Pl}}^{2}} .
$$

Here, $T=g^{\mu \nu} T_{\mu \nu}$ is the standard energy momentum tensor associated to (4). On the other hand, if we perform a small perturbation of the background metric $g_{\mu \nu}=$ $g_{0 \mu \nu}+2 \Phi \delta_{\mu}^{0} \delta_{\nu}^{0}$, we can write the linearized fourth order equation for the metric perturbation $\Phi$, and interpret this perturbation as the usual Newtonian potential. In [1], it was shown that we can recover an approximate Poisson equation and the NL if $\varepsilon_{0}^{\prime \prime}$ is small enough, i.e. at 
distances $r \gg\left(\varepsilon_{0}^{\prime \prime}\right)^{1 / 2}$. But in the SMGMs under study:

$$
\varepsilon^{\prime \prime}(R)=\lambda n(n-1)\left(R / \mu^{2}\right)^{n-1} / R .
$$

Models with $n<2(n \neq 0,1)$ have typically large values of $\varepsilon_{0}^{\prime \prime}$ due to their inverse dependence on the Hubble scale $R_{0} \sim H_{0}^{2}$.

On the other hand, in [10], the gravitational force which appears in the paradigmatic model $(n=-1)$ 2] due to a diffuse source in a locally de Sitter background was calculated. A linearly growing behavior was found, which is unacceptable because, for instance, it can increase the interaction between the Milky Way and Andromeda by six orders of magnitude [10]. To arrive at this conclusion, the authors also performed a similar expansion and analyzed the first order tensorial equation from (11) whose trace is given by (6).

Finally, in Reference 9], it was shown that these SMGMs are equivalent to Scalar-Tensor Theories (STTs) excluded by Solar System (SS) experiments. The MGM action leads to fourth order equations, which can be studied with the usual EEs if we add a new Scalar Degree of Freedom (SDF)-It is the so called Einstein frame [12]-. This new field has the following potential:

$$
V_{\varphi}=\frac{M_{\mathrm{P}}^{2}\left[R(\varphi) \varepsilon_{\varphi}^{\prime}-\varepsilon_{\varphi}\right]}{2\left[1+\varepsilon_{\varphi}^{\prime}\right]^{2}}
$$

where $R(\varphi)$ is the solution of the equation $\varepsilon^{\prime}(R)=$ $\exp \left(\sqrt{2 / 3} \varphi / M_{\mathrm{Pl}}\right)-1$, and $\varepsilon_{\varphi}^{(n)}=\varepsilon^{(n)}(R(\varphi))$. Its mass is related to the second derivative of the potential:

$$
m_{\varphi}^{2}=\frac{d^{2} V_{\varphi}}{d \varphi^{2}}=\frac{1}{3 \varepsilon_{\varphi}^{\prime \prime}}-\frac{R(\varphi)\left[3-\varepsilon_{\varphi}^{\prime}\right]+4 \varepsilon_{\varphi}}{3\left[1+\varepsilon_{\varphi}^{\prime}\right]^{2}} .
$$

In the vacuum solution, this field satisfies $R\left(\varphi_{0}\right)=R_{0} \sim$ $\mu^{2}$, which implies that the typical mass is of order $m_{\varphi} \sim$ $\mu$. However, if $m_{\varphi} \sim H_{0}<10^{-18} \mathrm{eV}$, the model can be excluded by SS tests, such as the deflection of light by the Sun, because $\varphi$ mediates a new force with a long range 9,13 .

In conclusion, several authors have detected important problems to the viability of SMGMs. Indeed, these three works are related because they have obtained inappropriate behaviors of the gravitational theory taking into account its vacuum state.

Different solutions have been proposed for some of these three problems [3]. For instance, Dick 11] has proposed a fine tuning to save the gravitational potential. A model that verifies $\varepsilon_{0}^{\prime \prime}=0$ has the correct NL, but even more significantly, this tuning resolves the two other problems: the usual gravitational interaction between galaxies is recovered, at least at the linearized equations; and the SDF has a divergent mass (9). Assuredly, the scalar field is not well defined on the vacuum state. This result can be understood because this field takes into account the new degree of freedom of the gravity due to its fourth order equations, but if $\varepsilon_{0}^{\prime \prime}=0$, the metric has associated the standard second order equations in vacuum.

A fine tuning usually means a physical misunderstanding, but there could be a fundamental reason for this cancellation. For instance, Dick has proposed the following example [1]]:

$$
\varepsilon(R)=-15 \mu^{4} / R+25 \mu^{6} / R^{2} .
$$

The vacuum solution reads $R_{0}=5 \mu^{2}$. These two terms have the same importance as the $\mathrm{EH}$ one in the vacuum state, which means that the gravitational coupling is modified. The effective $M_{\mathrm{Pl}}$ can be deduced from (6): $M_{\mathrm{eff}}^{2}=M_{\mathrm{Pl}}^{2}\left(1+\varepsilon_{0}^{\prime}\right)$, and in this particular model: $M_{\text {eff }}^{2}=5 M_{\mathrm{Pl}}^{2} / 6[11]$.

On the other hand, in Reference [14], it has been shown that we can recover Newton's Gravity Law (NGL) through the Schwarzschild solution inside a de Sitter (or anti-de Sitter) space. In fact, the metric:

$$
\begin{aligned}
d s^{2} & =-A(r) d t^{2}+A(r)^{-1} d r^{2}+r^{2} d \Omega^{2}, \\
A(r) & =1-\frac{2 m}{M_{\mathrm{Pl}}^{2} r}-\frac{R_{0} r^{2}}{12}
\end{aligned}
$$

is solution of the equations of motion (11) for a pointlike source of mass $m$ and with $R_{0}$ given by Equation (5). Therefore, we can argue that with a small enough background curvature any astrophysical test of gravity, which depends on the Schwarzschild solution and its NL, will be unaffected by the studied SMGMs.

However, this solution is not completely satisfactory because it seems in contradiction with the previous results. Furthermore, without a well defined NL, we can not identify the $M_{\mathrm{Pl}}$ in the action. In the same Reference [14], it has been commented that in the $\mu \rightarrow 0$ limit, we should recover the standard NL. Assuredly, if we remove the modification in the action, i.e. $\varepsilon(R) \rightarrow 0$, we recover the $\mathrm{EH}$ one. However, if we follow the arguments presented in 11], we arrive at the conclusion that the effective $M_{\mathrm{Pl}}$ is given by $5 M_{\mathrm{Pl}}^{2} / 6$ in the model (10) even in the $\mu \rightarrow 0$ limit. We can find similar surprises in the discussed results of [9, 10]. Without the tuning, the three problems present more inappropriate behaviors in the EH limit.

In our opinion, the question is that SMGMs modify the gravity at low energies. Therefore, it is consistent that the gravitational interaction was very different at low curvatures or low densities. This is the expected behavior, and the three discussed results agree with it showing that the gravitational interaction is more different for smaller values of $\mu$. The interesting question is in what kind of physical environments we can use their results to contrast with experiments or observations.

As it has been pointed out in 15, the expansions in the scalar curvature perturbations around the de Sitter vacuum solutions for the analyzed SMGMs (3) present 
the following form:

$$
\varepsilon(R)=\varepsilon_{0}\left[1+\mathcal{O}\left(\frac{\delta R}{R_{0}}\right)+\mathcal{O}\left(\frac{\delta R}{R_{0}}\right)^{2}+\ldots\right]
$$

The experiments which provide us with the most precise values of NGL and the $M_{\mathrm{Pl}}$ are realized on the Earth [16] with $\delta R / R_{0} \sim \mathcal{O}\left(\rho_{\text {Air }} / H_{0}^{2} M_{\mathrm{Pl}}^{2}\right) \sim 10^{27}$ (taking into account the air density $\rho_{\text {Air }} \sim 10^{15} \mathrm{eV}^{4}$ ), which means that we can not identify the $M_{\mathrm{Pl}}$ with the formula given in Reference 11]. The expansion can not be truncated inside the SS either; for example, we can roughly estimate the minimal SS curvature by the typical local Dark Matter $(\mathrm{DM})$ value $\rho_{\mathrm{DM}}^{\text {local }} \sim 10^{-1} \mathrm{GeV} / \mathrm{cm}^{3} \sim 10^{-6} \mathrm{eV}^{4}$ [17, which implies $\delta R / R_{0} \sim 10^{6}$.

Indeed, we can use this number as a typical value inside a galaxy. In such a case, we can not estimate the force between galaxies with the result reported in 10]. In fact, as their authors recognized, the linearized approximation around the vacuum solution inside the source (for example, the Milky Way) is not legitimate. Furthermore, the situation is analogous inside the body which can feel this possible force (such as Andromeda). Therefore, the correct metric solution of these non linear equations, which drives its dynamic, can differ very much from the result found in 10 .

Finally, in the equivalent STT, we can not use the vacuum values for the scalar field to study its effects in the SS [18. Without performing any calculations, we can guess that SMGMs could present a very different gravitational interaction close to the vacuum. The Reference [9] has shown it in a very elegant way, but the experiments or observations which are used to constrain STTs are not realized on vacuum. The SS tests restrict severely STTs if the force mediated by the new scalar field has associated a long range. However, as in the chameleon case 18, 19], the characteristics of this field depend significantly on the local curvature and matter content, to which it is strongly coupled. The situation is again tricky, and to illustrate it, we can estimate the range of this new force inside the Sun supposing a Yukawa-type potential exponentially suppressed by $m_{\varphi}$. If $R \gg \mu^{2}$, we find by using Equations (91) and (7):

$$
m_{\varphi}^{2}=\frac{d^{2} V_{\varphi}}{d \varphi^{2}} \simeq \frac{1}{3 \varepsilon_{\varphi}^{\prime \prime}}=\frac{\lambda R}{3 n(n-1)}\left(\frac{R}{\mu^{2}}\right)^{1-n}
$$

For instance, for the case $n=-1: m_{\varphi} \sim 10^{12} \mathrm{eV}$ $\left(\delta R / R_{0} \sim 10^{30}\right.$ with $\left.\rho_{\text {Sun }} \sim 10^{18} \mathrm{eV}^{4}\right)$, we observe that the range is of order of $m_{\varphi}^{-1} \sim 10^{-19} \mathrm{~m}$ and conclude that the scalar field can not produce a force with observable effects out of the Sun (with a radius of $r_{S} \sim 10^{9} \mathrm{~m}$ ). The situation is similar inside the Earth and all the typical
SS gravitational sources ${ }^{1}$.

The above estimations show that it is difficult to expect that the truncated expansions around the vacuum state were able to describe any physical observation. Practically, this type of expansions can only be linearized for cosmological studies and only in very recent (or future) times when $\delta R / R_{0} \sim \Omega_{\mathrm{M}} / \Omega_{\Lambda} \sim 0.3$ [20] (or smaller), but this is one of the most interesting features of SMGMs. They can modify the usual gravity at low curvatures and explain the present cosmology without a dark energy component.

If these models make any sense, they have to recover NGL at intermediate energies. We can not perform an expansion around the vacuum state, but we can perform an iterative method to resolve the Equation (11). The zero order should be given by $\operatorname{EEs}(\varepsilon(R) \rightarrow 0)$ :

$$
R_{\mathrm{E} \mu \nu}-\frac{1}{2} R_{\mathrm{E}} g_{\mathrm{E} \mu \nu}=\frac{T_{\mu \nu}}{M_{\mathrm{Pl}}^{2}} .
$$

Therefore, we find the usual relations: $R_{\mathrm{E}}=-T / M_{\mathrm{Pl}}^{2}$ and $R_{\mathrm{E} \mu \nu}=\left(T_{\mu \nu}-T g_{\mathrm{E} \mu \nu} / 2\right) / M_{\mathrm{Pl}}^{2}$. To obtain the first correction to EEs, we can interpret the new terms like a new source: $\varepsilon_{\mathrm{E}}^{(n)}=\varepsilon^{(n)}\left(-T / M_{\mathrm{Pl}}^{2}\right)$. Up to this first order, we can write:

$$
R_{1 \mu \nu}-\frac{1}{2} R_{1} g_{1 \mu \nu}=\frac{T_{\mu \nu}+T_{1 \mu \nu}}{M_{\mathrm{Pl}}^{2}},
$$

with

$$
\begin{aligned}
T_{1 \mu \nu} & =\frac{M_{\mathrm{Pl}}^{2} \varepsilon_{\mathrm{E}}+T \varepsilon_{\mathrm{E}}^{\prime}}{2} g_{\mathrm{E} \mu \nu}-\varepsilon_{\mathrm{E}}^{\prime} T_{\mu \nu} \\
& -M_{\mathrm{Pl}}^{2} \mathcal{I}_{\mathrm{E} \alpha \beta \mu \nu} \nabla_{\mathrm{E}}^{\alpha} \nabla_{\mathrm{E}}^{\beta} \varepsilon_{\mathrm{E}}^{\prime} .
\end{aligned}
$$

Here, the metric $g_{\mathrm{E} \mu \nu}$ is given by EEs (14) and $\mathcal{I}_{\mathrm{E} \alpha \beta \mu \nu} \equiv$ $\left(g_{\mathrm{E} \alpha \beta} g_{\mathrm{E} \mu \nu}-g_{\mathrm{E} \alpha \mu} g_{\mathrm{E} \beta \nu}\right)$. This procedure is inappropriate for conformal matter $(\mathrm{T}=0)$ inside the studied SMGMs (3), but it is very helpful for non relativistic matter without pressure, $T_{\mu \nu}=\rho \delta_{\mu}^{0} \delta_{\nu}^{0}$ :

$$
\begin{aligned}
T_{1 \mu \nu} & =\lambda M_{\mathrm{Pl}}^{2} \mu^{2}\left[\left(\frac{\rho}{M_{\mathrm{Pl}}^{2} \mu^{2}}\right)^{n}\left(\frac{1+n}{2} g_{\mathrm{E} \mu \nu}-n \delta_{\mu}^{0} \delta_{\nu}^{0}\right)\right. \\
& \left.-n \mu^{-2} \mathcal{I}_{\mathrm{E} \alpha \beta \mu \nu} \nabla_{\mathrm{E}}^{\alpha} \nabla_{\mathrm{E}}^{\beta}\left(\frac{\rho}{M_{\mathrm{Pl}}^{2} \mu^{2}}\right)^{n-1}\right] .
\end{aligned}
$$

Clearly, we do not find any modification to EEs for $\varepsilon(R)=0$. Indeed, for a small enough value of $\mu$, the correction is always negligible $(n<1)$. It is interesting to estimate when the maximum value of the new energy contribution $\left(|| T_{1 \mu \nu} \| \equiv \operatorname{Max}\left|T_{1 \mu \nu}\right|\right)$ is much smaller than $\rho$, which implies that NGL is recovered. For instance, if

\footnotetext{
${ }^{1}$ It is interesting to note that we find a negative square-mass term for the model with $\lambda=n=-1$ [2] reproducing the instabilities studied in 8] from an independent approach.
} 
we can neglect the temporal and spatial variations of the source, we find the following relation:

$$
\left\|T_{1 \mu \nu}\right\| \ll\left\|T_{\mu \nu}\right\| \Rightarrow \rho \gg \mu^{2} M_{\mathrm{Pl}}^{2}, \quad(n<1) .
$$

We observe that the same estimations, which were used to show that the vacuum expansions were incorrectly truncated, allow us to conclude that we have a well defined NL inside the SS for the proposed models with $\mu \sim H_{0}(n<1)$. In fact, the two approximations are opposite in certain sense. This NL works for large densities (or curvatures) in relation to the typical cosmological constant scale: $M_{\mathrm{Pl}}^{2} H_{0}^{2} \sim 10^{-12} \mathrm{eV}^{4}\left(H_{0}^{2} \sim 10^{-66} \mathrm{eV}^{2}\right)$, whereas the linearized vacuum expansions work for small densities (or curvatures) with respect to the same scale.

It is fair to say that we are not able to assure NGL for the interesting physical environment studied in [10]. The Condition (18) can not be satisfied in intergalactic spaces for $\mu \sim H_{0}$. Indeed, we think that this is another very interesting property of SMGMs. They modify NGL at low densities, which is the expected behavior for $n<1$. For example, if we neglect the mentioned temporal and spatial variations of the source, we obtain the following correction for the model with $n=-1$ [2]:

$$
T_{1 \mu \nu}=\lambda \frac{\mu^{4} M_{\mathrm{Pl}}^{4}}{\rho} \delta_{\mu}^{0} \delta_{\nu}^{0} .
$$

From this point of view, we have a new source of gravitation, which is certainly a DM source since we can only detect an anomalous metric behavior. It may be interesting to try to explain galactic dynamic anomalies or even rotation curves without DM halos, which requires the introduction of new unobserved particles in the standard framework 21]. Similar approaches to general MGMs can be found in previous works with promising results [4]. However, if $\left\|T_{1 \mu \nu}\right\|>\left\|T_{\mu \nu}\right\|$, higher order corrections have to be taken into account and a numerical calculation seems necessary. In this sense, we propose an iterative method by repeating the described procedure up to an eventual convergence. Alternatively, if we know the metric, we can infer the necessary source. If this source does not agree with the observed one, we can deduce a possible function $\varepsilon(R)$ that can explain the difference. For simplicity, in a first step, we can study the functions given by Equation (3) parameterized by $\mu, n$ and $\lambda$.

Before concluding this article, we would like to comment briefly about SMGMs inside the Palatini formalism, in which the metric and the connection are taken as independent variables [6]. We think that our discussion can also clarify several aspects concerning this approach. For instance, it is possible to find in the literature different works with different results about the NL in this formulation [15, 22]. In our opinion, it is fundamental to work at intermediate energies, as it has been performed very recently in [15], and not in the traditional low density approximation.
We have studied the viability of SMGMs, which could be interesting to explain not only the present acceleration of the Universe but also its DM content. The idea is that they deviate from GR at low energies or low curvatures. In contrast, we recover the NL at intermediate scales. More analyses to clarify the possible existence of other kinds of problems have to be performed before postulating them as a serious alternative. Work is in progress in this direction.

Acknowledgments - We thank A. Rajaraman and F. Takayama for important comments. This Work is supported in part by NSF PHY-0239817 grant, BFM 200201003 project (DGICYT) and Fulbright-MEC program.

[1] A.A. Starobinsky, Phys. Lett. B 91 (1980) 99; A. Dobado and A. Lopez, Phys. Rev. D 52 (1995) 1895

[2] S. M. Carroll et al., Phys. Rev. D 70 (2004) 043528

[3] S. Nojiri and S.D. Odintsov, Phys. Rev. D 68 (2003) 123512, Gen. Rel. Grav. 36 (2004) 1765 and Mod. Phys. Lett. A 19 (2004) 627

[4] S. Capozziello et al., astro-ph/0303041 Phys. Rev. D 71 (2005) 043503; gr-qc/0410046 Int. J. Mod. Phys. D 12 (2003) 1969 and Phys. Lett. A 326 (2004) 292

[5] G.J. Olmo, gr-qc/0505135 and gr-qc/0505101 I. Navarro and K. Van Acoleyen, gr-qc/0506096 J.A.R. Cembranos, in preparation

[6] D. Vollick, Class. Quant. Grav. 21 (2004) 3813; E. Flanagan, Class. Quant. Grav. 21 (2004) 3817; G. Allemandi et al., Phys. Rev. D 70 (2004) 043524; P. Wang and X. Meng, Phys. Lett. B 584 (2004) 1

[7] S. Nojiri and S.D. Odintsov, Phys. Lett. B 576 (2003) 5

[8] A. Dolgov and M. Kawasaki, Phys. Lett. B 573 (2003) 1

[9] T. Chiba, Phys. Lett. B 575 (2003) 1

[10] M.E. Soussa and R.P. Woodard, Gen. Rel. Grav. 36 (2004) 855

[11] R. Dick, Gen. Rel. Grav. 36 (2004) 217

[12] G. Magnano and L.M. Sokolowski, Phys. Rev. D 50 (1994) 5039

[13] C.M. Will, Living Rev. Rel. 4 (2001) 4

[14] A. Rajaraman, astro-ph/0311160

[15] T.P. Sotiriou, gr-qc/0507027

[16] J.H. Gundlach and S.M. Merkowitz, Phys. Rev. Lett. 85 (2000) 2869

[17] S. Eidelman et al. Phys. Lett. B 592 (2004) 1.

[18] J.A.R. Cembranos et al., in progress

[19] J. Khoury and A. Weltman, Phys. Rev. Lett. 93 (2004) 171104 and Phys. Rev. D 69 (2004) 044026

[20] D. N. Spergel et al., Astrophys. J. Suppl. 148 (2003) 175

[21] J.R. Ellis et al., Nucl. Phys. B 238 (1984) 453; L. Covi et al., Phys. Rev. Lett. 82 (1999) 4180; G. Servant and T.M.P. Tait, Nucl. Phys. B 650 (2003) 391; H.C. Cheng et al., Phys. Rev. Lett. 89 (2002) 211301; J.A.R. Cembranos et al., Phys. Rev. Lett. 90 (2003) 241301, hep-ph/0507066 and Phys. Rev. D 68 (2003) 103505; J.L. Feng et al., Phys. Rev. Lett. 91 (2003) 011302 and Phys. Rev. D 68 (2003) 063504

[22] A. Dominguez and D. Barraco, Phys.Rev. D 70 (2004) 043505; X.H. Meng and P. Wang, Gen. Rel. Grav. 36 (2004) 1947; G.J. Olmo, gr-qc/0505136 